
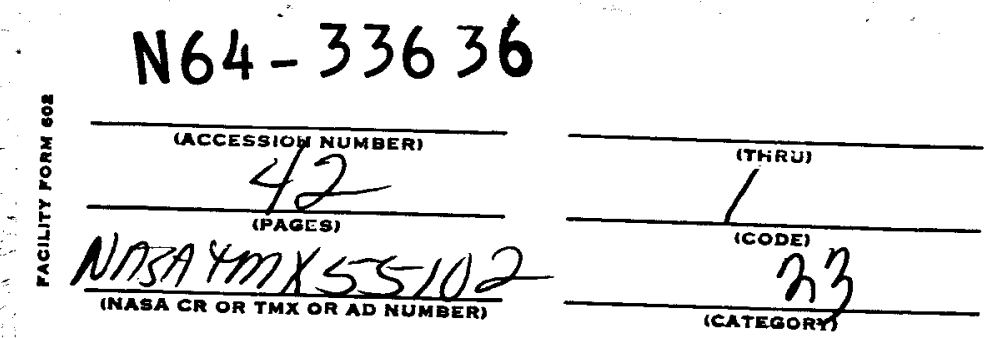

\title{
DECOMPOSITION \\ OF THE SCHRÖDINGER EQUATION \\ FOR TWO IDENTICAL PARTICLES \\ AND A THIRD PARTICLE OF FINITE MASS
}

$\frac{0}{0}$

\author{
BY \\ A. K. BHATIA
}

A. TEMKIN

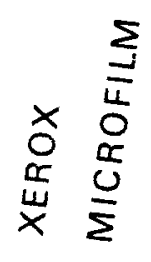

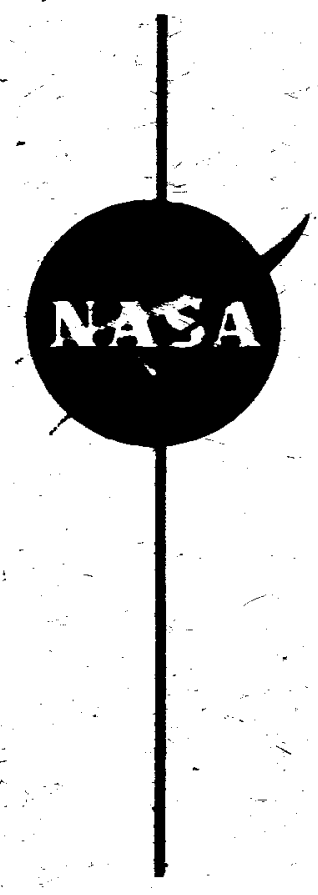

SEPTEMBER 1964

GODDARD SPACE FLIGHT CENTER GREENBELT, MARYLAND 
Decomposition of the Schrödinger Equation for

Two Ideatical Particles and a Third Particle of

Finite Mass

\begin{abstract}
A. K. Bhatia ${ }^{\dagger}$ and A. Temkin
Theoreticil Division

Goddard Space Flight Center

National Aeronautics and Space Administration

Greenbelt, Maryland
\end{abstract}

An angular momentum decomposition of the Schrodinger equation is extended to the case noted in the title. (The case of three unequal mass particles is treated in an appendix.) The decomposition is effected with the use of a symmetric choice of Euler angles, and the radial equations are given in two use ful forms. The radial equations are shown to yield the Born-Oppenheimer equations for $\mathrm{H}_{2}{ }^{+}$in the limit that the two identical particles approach infinite mass. Other aspects of this Iimit are discussed, and general rules which relate the total angular momentum states of the three-body system with the molecular states of $\mathrm{H}_{2}{ }^{+}$are examined.

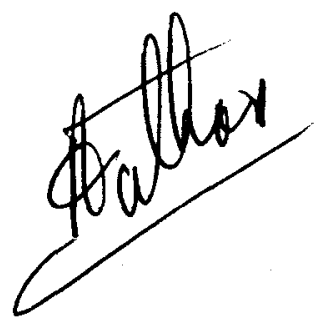




\section{INIRODUCTION}

In a previous review ${ }^{1}$ we have presented a detailed investigation of the decomposition of the Schrbdinger equation for two identical particles, obeying the exclusion principle, in the central field of an infinitely heavy center of force. This, of course, is the prototype of the two-electron atom. The major correction to this idealization is the recoil effects of the nucleus. It is the primary purpose of this paper ti give the extension of the above decomposition to include the finite mass of the previously assumed fixed center. (The changes necessary in the radial equations for three unequal mass particles are given in Appendix B.)

The resulting decomposition is still sufficiently general to allow an arbitrary mass to the identical particles as well as the nonidentical third particle. As a rather different limit of this system from the previous one, one can consider the limit in which the two identical particles become infinitely heavy and the third particle assumes the electron mass. This corresponds to the $\mathrm{H}_{2}{ }^{+}$ limit, and it is of considerable interest to see how the radial equations and the three-body symmetries go over into the equations and symmetries of the Born-Oppenheimer approximation in that case.

This is particularly relevant at the present time since the absorption of $\mu$ mesons in hydrogen ${ }^{2}$ via the molecule $(p-\mu-p)$ can presumably only be explained by the deviations of this $\mu$ mesic counterpart of $\mathrm{H}_{2}{ }^{+}$from the Born-Oppenheimer approximation ${ }^{3}$. The $\mathrm{H}_{2}{ }^{+}$limit will be the subject of Section III. 
II KINFTIC ENERGY AND RADIAL EQUATIONS

Let $\rho_{1}$ and $\rho_{2}$ be the coordinates of two identical particles, each of mass $m$, in an arbitrary fixed coordinate system. Let

$P_{3}$ be the coordinates of a third particle of mass $M$. The kinetic energy can be written:

$$
T=-\frac{1}{2 m}\left(\nabla_{\rho_{1}}^{2}+\nabla_{\rho_{2}}^{2}\right)-\frac{1}{2 M} \nabla_{\rho_{2}}^{2}
$$

With the introduction of the difference vectors

$$
\begin{aligned}
& r_{1}=p_{1}-P_{3} \\
& r_{2}=P_{2}-P_{2}
\end{aligned}
$$

and the center of mass coordinate $\underset{\sim}{\mathrm{R}}$ :

$$
R=\frac{m\left(p_{1}+p_{2}\right)+M p_{3}}{2 m+M}
$$


the kinetic energy becomes

$$
T=-\frac{1}{2 \mu}\left(\nabla_{r_{1}}^{2}+\nabla_{r_{2}}^{2}\right)-\frac{1}{M} \nabla_{r_{1}} \cdot \nabla_{r_{2}}-\frac{1}{2(2 m+M)} \nabla_{R}^{2},
$$

where

$$
\mu=\frac{m M}{m+M} \text {. }
$$

The last term in $(2.38)$ is clearly the kinetic energy of the center of mass. In any closed system the potentlal will be independent of $\underset{\sim}{R}$, so that the effect of this term will be to subtract a center of mass energy, $E_{C M}$, from the total energy, $E_{T}$, to give an effective energy $\mathrm{E}$ for a Schrödinger equation with the last term in (2.3a) absent. Thus we can replace the kinetic energy by an effective kinetic energy governing the internal motion

$$
T_{e}=-\frac{1}{2 \mu}\left(\nabla_{r_{1}}^{2}+\nabla_{r_{2}}^{2}\right)-\frac{1}{M} \nabla_{r_{1}} \cdot \nabla_{r_{2}},
$$


to be used with an effective energy

$$
E=E_{T}-E_{C M}
$$

$\mathrm{T}_{\mathrm{e}}$ now differs in form from the kinetic energy of the twoelectron fixed nucleus problem ${ }^{1}$ by the addition of the final cross term. This additional term is the well-known mass polarization term which in most helium applications is treated in perturbation theory (which is quite adequate for the present experimental accuracy). We shall include the effect of this term exactly.

It will be recalled from I that the major task in effectliag the decomposition is to find the kinetic energy in terms of the Euler angles and residual coordinates in place of the particle coordinates. We introduce formally the same Euler angles as in I; namely $\theta$ the angle between the space-flxed $\hat{z}$ direction and $\hat{r}_{1} \times \hat{r}_{?} \propto \hat{z}^{\prime}, \Phi$ the angle between the space-flxed $\hat{\mathbf{x}}$ direction, and $\hat{z} \times \hat{z}^{\prime}-\hat{x}^{\prime}$, and $\Psi$ the angle between $\hat{x}^{\prime}$ and $\left(\hat{r}_{2}-\hat{r}_{1}\right)$. Now, since the form of $\nabla_{r_{1}}^{2}+\nabla_{r_{2}}^{2}$ is the same as in $I$, the transformation of those terms in terms of the three Euler angles and three residual coordinates can simply be taken over from $I$. We need only consider therefore the remalning cross term, and since we handle it in a very similar manner, we shall be mercifully brief. In spherical coordinates the cross term is

$$
\nabla_{n_{1}} \cdot \nabla_{r_{2}}=\cos \theta_{12} \frac{\partial^{2}}{\partial r_{1} \partial r_{2}}+\left\{\frac{1}{r_{1}}\left(u \frac{\partial^{2}}{\partial r_{2} \partial v_{1}}+v \frac{\partial^{2}}{\partial r_{2} \partial \varphi_{1}}\right)+\left(1 \vec{z}^{2}\right)\right\}
$$


$+\frac{1}{x_{1} x_{2}}\left\{a \frac{\partial^{2}}{\partial v_{1} \partial v_{2}}+b \frac{\partial^{2}}{\partial \phi_{1} \partial \varphi_{2}}+\left[c \frac{\partial^{2}}{\partial v_{1} \partial \phi_{2}}+\left(1 \vec{\rightleftarrows}^{2}\right)\right]\right\}$

where

$u=\sin v_{2} \cos v_{1} \cos \left(\phi_{2}-\phi_{1}\right)-\cos v_{2} \sin v_{1}$

$v=\frac{\sin v_{2}}{\sin v_{1}} \sin \left(\phi_{2}-\phi_{1}\right)$

$a=\cos v_{1} \cos v_{2} \cos \left(\varphi_{2}-\varphi_{1}\right)+\sin v_{1} \sin v_{2}$

$b=\frac{\cos \left(\phi_{2}-\phi_{1}\right)}{\sin \nu_{1} \sin \nu_{2}}$

$c=\frac{\cos v_{1}}{\sin v_{2}} \sin \left(\phi_{1}-\phi_{2}\right)$

When the residual coordinates are taken as $r_{1}, r_{2}, \theta_{12}$, then the 
transformation only involves first and second partial derivatives of $v_{1}, q_{1}, v_{2}, \phi_{2} \quad$ with respect to $\theta, \Phi, \Psi, \theta_{12}$. (The latter are referred to as $x_{\alpha}, \alpha=1 \ldots 4$ below). The cross term can therefore be written

$$
\begin{aligned}
& \nabla_{n_{1}} \cdot \nabla_{\alpha_{2}}=\cos \theta_{12} \frac{\partial^{2}}{\partial r_{1} \partial r_{2}}+\sum_{\alpha=1}^{4}\left[\frac{1}{z_{1}}\left(u \frac{\partial x_{\alpha}}{\partial \theta_{1}}+v \frac{\partial x_{\alpha}}{\partial \varphi_{1}}\right) \frac{\partial^{2}}{\partial x_{\alpha} \partial r_{2}}\right. \\
& +(1 \overrightarrow{2})]+\frac{1}{k_{1} \varepsilon_{2}}\left\{\sum _ { \alpha = 1 } ^ { 4 } \left[a \frac{\partial x_{\alpha}}{\partial x_{1}} \frac{\partial x_{\alpha}}{\partial v_{2}}+\operatorname{lo} \frac{\partial x_{\alpha}}{\partial q_{1}} \frac{\partial x_{\alpha}}{\partial \varphi_{2}}\right.\right. \\
& \left.+\left[c \frac{\partial x_{\alpha}}{\partial v_{1}} \frac{\partial x_{\alpha}}{\partial \varphi_{2}}+1 \rightleftarrows^{2}\right)\right] \frac{\partial^{2}}{\partial x_{\alpha}^{2}}+\sum_{\alpha=1}^{4}\left[a \frac{\partial^{2} x_{\alpha}}{\partial v_{1} \partial v_{2}}\right. \\
& \left.+b \frac{\partial^{2} x_{\alpha}}{\partial \varphi_{1} \partial \varphi_{2}}+\left(c \frac{\partial^{2} x_{\alpha}}{\partial v_{1} \partial \varphi_{2}}+\left(1 \vec{\rightleftarrows}^{2}\right)\right)\right] \frac{\partial}{\partial x_{\alpha}} \\
& +\sum_{\alpha} \sum_{\beta=1}^{4}\left[a\left\{\frac{\partial x_{\alpha}}{\partial v_{1}} \frac{\partial x_{\beta}}{\partial v_{2}}+\left(1 \vec{z}^{2}\right)\right\}+k\left\{\frac{\partial x_{\alpha}}{\partial \phi_{1}} \frac{\partial x_{B}}{\partial \phi_{2}}\right.\right. \\
& \left.+(1 \rightleftarrows 2)\}+\left\{c\left(\frac{\partial x_{\alpha}}{\partial v_{1}} \frac{\partial x_{\beta}}{\partial \varphi_{2}}+\frac{\partial x_{\alpha}}{\partial \varphi_{2}} \frac{\partial x_{\beta}}{\partial v_{1}}\right)+\left(1 \overrightarrow{2}^{2}\right)\right\}\right] \frac{\partial^{2}}{\partial x_{\alpha} \partial x_{\beta}}
\end{aligned}
$$


$-8-$

The results in the form of the coefficients of the derivatives with respect to $x_{\alpha}$ expressed as functions of $\theta, \Phi, \psi, \theta_{12}$ are given in Table I. Finally we can write the cross term as an operator whose effect on the vector spherical harmonics have been fully explored in $I$.

$$
\begin{aligned}
& \nabla_{r_{1}} \cdot \nabla_{r_{2}}=\cos \theta_{12} \frac{\partial^{2}}{\partial r_{1} \partial r_{2}}-\frac{\sin \theta_{12}}{r_{2}} \frac{\partial}{\partial r_{1}}\left(\frac{\partial}{\partial \theta_{12}}+\frac{1}{2} \frac{\partial}{\partial \psi}\right) \\
& -\frac{\sin \theta_{12}}{r_{1}} \frac{\partial}{\partial r_{2}}\left(\frac{\partial}{\partial \theta_{12}}-\frac{1}{2} \frac{\partial}{\partial \psi}\right) \\
& +\frac{1}{r_{1} x_{2}}\left[-\frac{1}{\sin \theta_{12}} \frac{\partial}{\partial \theta_{12}}-\cos \theta_{12} \frac{\partial^{2}}{\partial \theta_{12}^{2}}+\frac{\cos \theta_{12}}{4} \frac{\partial^{2}}{\partial \psi^{2}}\right. \\
& -\frac{1}{2 \sin ^{2} \theta_{12}}\left(\sin 2 \psi \Lambda_{2}-\cos 24 \Lambda_{1}\right) \\
& \left.+\frac{\cos \theta_{12}}{2 \sin ^{2} \theta_{12}}\left(\frac{\partial^{2}}{\partial \psi^{2}}+\frac{M^{2}}{\hbar^{2}}\right)\right]
\end{aligned}
$$

$(2.13)$ 
$-9-$

angular momentum squared operator, (I4I), in terms of the Euler angles.

The wave function is now expanded in the form (I55)

$$
\begin{aligned}
\Psi_{l m}\left(\underline{s}_{1}, r_{2}\right)= & \sum_{x}^{\prime \prime}\left[f_{l}^{x+}\left(r_{1}, r_{2}, \theta_{12}\right) D_{l}^{(m, x)+}(\theta, \Phi, \psi)\right. \\
& \left.+f_{l}^{x-}\left(r_{1}, r_{2}, \theta_{12}\right) g_{l}^{(m, x)-}(\theta, \Phi, \psi)\right]
\end{aligned}
$$

$(2.14)$

where $D_{l}^{(m, x) \pm}$ are the exchange vector spherical harmonics (I54), the simultaneous eigenfunction of $\underline{M}^{2}, M_{z}$ and $\varepsilon_{12}$. The operator

$\nabla_{t_{1}} \cdot \nabla_{n_{2}}$ as expressed (2.13) is now such that its operation on these $g_{l}^{(m, x) \pm}$ functions can readily be found from $(I 65,66)$. Thus the radial equations can simply be derived as an addition of terms to what was derived in I. Below we shall give the equations in two forms; the first in terms of simultaneous coupled equations for the "radial" functions $f_{l}^{x \pm}\left(r_{1}, x_{2}, \theta_{12}\right)$ :

$$
\left[L \theta_{12}+\frac{2 \mu}{\hbar^{2}}(E-V)\right] f_{l}^{x+}-\left(\frac{1}{r_{1}^{2}}+\frac{1}{r_{2}^{2}}\right)\left[\left\{\frac{l(l+1)-x^{2}}{2 \sin ^{2} \theta_{12}}+\frac{x^{2}}{4}\right.\right.
$$

$$
\begin{aligned}
& \left.\left.\because-\frac{\cot \theta_{12}}{4 \sin \theta_{12}} l(l+1) \delta_{1 x}\right\} f_{l}^{x+}+\frac{\cot \theta_{12}}{4 \sin \theta_{12}}\left\{B_{l}^{x+2} f_{l}^{(x+2)+}+\left(1-\delta_{\theta x}-\delta_{1 x}+\delta_{2 x}\right) B_{l x} f_{l}^{(x-2)+}\right\}\right] \\
& \left.+\frac{1}{r_{1}^{2}}-\frac{1}{\varepsilon_{2}^{2}}\right)\left[-\left\{n\left(\frac{1}{2} \cot \theta_{12}+\frac{\partial}{\partial \theta_{12}}\right)+\frac{e(l+1) \delta_{1 x}}{4 \sin \theta_{12}}\right\} f_{l}^{x-}\right. \\
& \left.+\frac{1}{4 \sin \theta_{12}}\left\{B_{l}^{x+2} f_{l}^{(x+2)-}-\left(1-\delta_{0 x}-\delta_{1 x}-\delta_{2 x}\right) B_{l x} f_{l}^{(n-2)-}\right\}\right] \\
& +\frac{2 \mu}{M} \frac{1}{r_{1} r_{2}}\left[\left\{\frac{\cos \theta_{12}}{2 \sin ^{2} \theta_{12}}\left(l(l+1)-x^{2}\right)-\frac{x^{2}}{4} \cos \theta_{12}-\frac{l(l+1)}{4 \sin ^{2} \theta_{12}} \delta_{1 x}\right\} f_{l}^{x+}\right.
\end{aligned}
$$


$-10-$

$$
\begin{aligned}
& \left.+\frac{1}{4 \sin ^{2} \theta_{12}}\left\{B_{l}^{x+2} f_{l}^{(x+2)+}+\left(1-\delta_{0 x}-\delta_{1 x}+\delta_{2 x}\right) B_{l x} f_{l}^{(x-2)+}\right\}\right] \\
& +\frac{2 \mu}{M}\left[\left\{\cos \theta_{12} \frac{\partial^{2}}{\partial r_{1} \partial r_{2}}-\frac{\sin \theta_{12}}{r_{2}} \frac{\partial^{2}}{\partial r_{1} \partial \theta_{12}}-\frac{\sin \theta_{12}}{r_{1}} \frac{\partial^{2}}{\partial r_{2} \partial \theta_{12}}-\frac{1}{r_{1} r_{2} \sin \theta_{12}} \frac{\partial}{\partial \theta_{12}}\right.\right. \\
& \left.\left.-\frac{\cos \theta_{12}}{r_{1} r_{2}} \frac{\partial^{2}}{\partial \theta_{12}^{2}}\right\} f_{l}^{x+}+\frac{x}{2} \sin \theta_{12}\left(\frac{1}{r_{1}} \frac{\partial}{\partial r_{2}}-\frac{1}{r_{2}} \frac{\partial}{\partial r_{1}}\right) f_{l}^{x-}\right]=0 \\
& {\left[L_{\theta_{12}}+\frac{2 \mu}{\hbar^{2}}(E-V)\right] f_{l}^{x-}-\left(\frac{1}{r_{1}^{2}}+\frac{1}{\varepsilon_{2}^{2}}\right)\left[\left\{\frac{l(l+1)-x^{2}}{2 \sin ^{2} \theta_{12}}+\frac{x^{2}}{4}+\frac{\cot \theta_{12}}{4 \sin \theta_{12}} \ell(l+1) \delta_{1 x}\right\} f_{l}^{x-}\right.} \\
& \left.+\frac{\cot \theta_{12}}{4 \sin \theta_{12}}\left\{\left(1-\delta_{0 x}\right) B_{l}^{x+2} f_{l}^{(x+2)-}+\left(1-\delta_{0 x}-\delta_{1 x}-\delta_{2 x}\right) B_{l x} f_{l}^{(x-2)-}\right\}\right] \\
& +\left(\frac{1}{z_{1}^{2}}-\frac{1}{s_{2}^{2}}\right)\left[\left\{x\left(\frac{1}{2} \cot \theta_{12}+\frac{\partial}{\partial \theta_{12}}\right)-\frac{l(l+1)}{4 \sin \theta_{12}} \sin _{12}\right\} f_{l}^{x+}\right. \\
& \left.-\frac{1}{4 \sin \theta_{12}}\left\{\left(1-\delta_{0 x}\right) B_{l}^{x+2} f_{l}^{(x+2)+}-\left(1-\delta_{0 x}-\delta_{1 x}+\delta_{2 x}\right) B_{l x} f_{l}^{(x-2)+}\right\}\right] \\
& +\frac{2 \mu}{M} \frac{1}{z_{1} n_{2}}\left[\left\{\frac{\cos \theta_{12}}{2 \sin ^{2} \theta_{12}}\left(l(l+1)-x^{2}\right)-\frac{x^{2}}{4} \cos \theta_{12}+\frac{l(l+1) \delta_{1 x}}{4 \sin ^{2} \theta_{12}}\right\} f_{l}^{x-}\right. \\
& \left.+\frac{1}{4 \sin ^{2} \theta_{12}}\left\{\left(1-\delta_{0 x}\right) B_{l}^{x+2} f_{\rho}^{(x+2)-}+\left(1-\delta_{0 x}-\delta_{1 x}-\delta_{2 x}\right) B_{\ell x} f_{l}^{(x-2)-}\right\}\right] \\
& +\frac{2 \mu}{M}\left[\left\{\cos \theta_{12} \frac{\partial^{2}}{\partial r_{1} \partial r_{2}}-\frac{\sin \theta_{12}}{r_{2}} \frac{\partial^{2}}{\partial r_{1} \partial \theta_{12}}-\frac{\sin \theta_{12}}{r_{1}} \frac{\partial^{2}}{\partial r_{2} \partial \theta_{12}}-\frac{1}{r_{1} r_{2}} \sin \theta_{12} \frac{\partial}{\partial \theta_{12}}\right.\right. \\
& \left.\left.-\frac{\cos \theta_{12}}{r_{1} r_{2}} \frac{\partial^{2}}{\partial \theta_{12}^{2}}\right\} f_{l}^{x-}+\frac{x}{2} \sin \theta_{12}\left(\frac{1}{r_{2}} \frac{\partial}{\partial r_{1}}-\frac{1}{r_{1}} \frac{\partial}{\partial r_{2}}\right) f_{l}^{x+}\right]=0
\end{aligned}
$$


The numbers $B_{\ell} x$ and $B_{\ell}^{x}$ are defined in (I68), and $S$-wave operator $\mathrm{I}_{\Theta_{12}}$ in (I7I). The fact that the equations are in this first form are coupled for a given $n$ implies that we are dealing with symmetric and antisymetric functions

$$
\begin{aligned}
& f_{l}^{x+}\left(r_{1}, r_{2}, \theta_{12}\right)= \pm(-1)^{l+x} f_{l}^{x+}\left(r_{2}, r_{1}, \theta_{12}\right) \\
& f_{l}^{x-}\left(r_{1}, r_{2}, \theta_{12}\right)= \pm(-1)^{2+x+1} f_{l}^{x-}\left(r_{2}, r_{1}, \theta_{12}\right)
\end{aligned}
$$

where the upper sign refers to the totally space symmetric wave function (singlet) and the lower, the space antisymmetric solution (triplet). The (anti) symmetry means that we can confine the solution to half the $r_{1}-r_{2}$ plane with an appropriate vanishing of the function or its normal derivative along $r_{1}=r_{2}$ (cf. Eqs. (I72) and (I73)). The second form of these equations that we shall give involves the "radial" coordinates $r_{I}, r_{2}, r_{12}$ and involves the asymmetric functions $\mathrm{F}_{l}^{x}$ and $\tilde{F}_{e}^{x}$.

$$
F_{l}^{n}\left(r_{1}, r_{2}, r_{12}\right)=f_{l}^{x+}\left(r_{1}, r_{2}, \theta_{12}\right)+f_{l}^{x-}\left(r_{1}, r_{2}, \theta_{12}\right)
$$




$$
\tilde{F}_{l}^{x}\left(r_{1}, r_{2}, r_{12}\right)=f_{l}^{x t}\left(r_{1}, r_{2}, \theta_{12}\right)-f_{l}^{x-}\left(r_{1}, r_{2}, \theta_{12}\right)
$$

where, on the rhs, $\theta_{12}$ is understood to be expressed as a function of $r_{1}, r_{2}, r_{12}$ through the law of cosines. By virtue of (2.16) we have

$$
\tilde{F}_{\ell}^{x}\left(r_{2}, r_{1}, r_{2}\right)= \pm(-1)^{\ell+x} F_{l}^{x}\left(r_{1}, r_{2}, r_{12}\right) .
$$

This relation enables us to write the equations in an uncoupled form for a given $x$ (the various $x$ 's are of course coupled to each other) but in a domain covering the whole $r_{2}-r_{2}$ plane. It is also convenient in writing this second form to combine some of the terms multiplied by $2 \cdot \mu / M$ in $(2.15)$ with $L_{r_{12}}$ (given In (I81)) to form a new S-wave operator $L^{\prime}{ }_{r_{12}}$ :

$$
\begin{aligned}
L_{r_{12}}^{\prime}= & \frac{1}{r_{1}} \frac{\partial^{2}}{\partial r_{1}^{2}} r_{1}+\frac{1}{r_{2}} \frac{\partial^{2}}{\partial r_{2}^{2}} r_{2}+\frac{r}{m}\left[\frac{2}{r_{12}} \frac{\partial^{2}}{\partial r_{12}^{2}} r_{12}\right. \\
& \left.+\frac{r_{1}^{2}+r_{12}^{2}-r_{2}^{2}}{r_{1} r_{12}} \frac{\partial^{2}}{\partial r_{1} \partial r_{12}}+\frac{r_{2}^{2}+r_{12}^{2}-r_{1}^{2}}{r_{2} r_{12}} \frac{\partial^{2}}{\partial r_{2}} \partial r_{12}\right]+\frac{\mu}{M} \frac{r_{1}^{2}+r_{2}^{2}-r_{12}^{2}}{r_{1} r_{2}} \frac{\partial^{2}}{\partial r_{1} \partial r_{2}}
\end{aligned}
$$

We also use the quantity $\rho$ 
$-1 j-$

$\therefore \quad p=\left[-r_{12}^{4}-\left(r_{1}^{2}-r_{2}^{2}\right)^{2}+2 r_{12}^{2}\left(r_{1}^{2}+r_{2}^{2}\right)\right]^{\frac{1}{2}}$

The equations are:

$$
\begin{aligned}
& {\left[L_{n+2}^{\prime}+\frac{2 \mu}{\hbar^{2}}(E-V)\right] F_{l}^{x}-\left(\frac{1}{z_{1}^{2}}+\frac{1}{z_{2}^{2}}\right)\left[\left\{\left(l(l+1)-x^{2}\right) \frac{2 n_{1}^{2} r_{2}^{2}}{\rho^{2}}+\frac{n^{2}}{4}\right\} F_{l}^{x}\right.} \\
& -l(l+1)\left(r_{1}^{2}+r_{2}^{2}-r_{12}^{2}\right) \frac{r_{1} r_{2}}{2 p^{2}} \delta_{1 x} \tilde{F}_{l}^{x}+B_{l}^{n+2}\left(r_{1}^{2}+r_{2}^{2}-r_{12}^{2}\right) \frac{r_{1} r_{2}}{2 p^{2}}\left\{F_{l}^{x+2}\right. \\
& \left.-\frac{\delta_{0 x}}{2}\left(F_{l}^{x+2}-\tilde{F}_{l}^{x+2}\right)\right\}+B_{l} x\left(r_{1}^{2}+r_{2}^{2}-r_{12}^{2}\right) \frac{r_{1} r_{2}}{2 p^{2}}\left(1-\delta_{0 x}-\delta_{1 x}\right)\left\{F_{l}^{x-2}\right. \\
& \left.\left.+\delta_{2 x} \tilde{F}_{l}^{n-2}\right\}\right]+\left(\frac{1}{r_{1}^{2}}-\frac{1}{r_{2}^{2}}\right)\left[\frac { n } { 2 } \left\{\frac{r_{1}^{2}+r_{2}^{2}-r_{12}^{2}}{\rho}\right.\right. \\
& \left.+\frac{\mu}{m} \frac{\rho}{r_{12}} \frac{\partial}{\partial r_{12}}\right\} \tilde{F}_{\ell}^{n}-\frac{\ell(\ell+1)}{2} \frac{r_{1} r_{2}}{\rho} S_{1 n} F_{q}^{x}+B_{l}^{x+2} \frac{r_{1} r_{2}}{2 \rho}\left\{-\tilde{F}_{l}^{x+2}\right. \\
& \left.\left.+\frac{\delta_{0 x}}{2}\left(F_{l}^{x+2}+\tilde{F}_{l}^{x+2}\right)\right\}+B_{l x} \frac{r_{1} r_{2}}{2 \rho}\left(1-\delta_{0 x}-\delta_{1 x}\right)\left\{\tilde{F}_{l}^{x-2}+\delta_{2 x} F_{l}^{x-2}\right\}\right] \\
& +\quad \frac{2 \mu}{M}\left[\left(r_{1}^{2}+r_{2}^{2}-r_{12}^{2}\right)\left\{\frac{l(l+1)-x^{2}}{\rho^{2}}-\frac{n^{2}}{8 z_{1}^{2} r_{2}^{2}}\right\} F_{l}^{x}\right. \\
& -e(l+1) \frac{r_{1} r_{2}}{\rho^{2}} \sin \tilde{F}_{l}^{x}+\frac{n p}{4 r_{1} r_{2}}\left(\frac{1}{r_{2}} \frac{\partial}{\partial r_{1}}-\frac{1}{r_{1}} \frac{\partial}{\partial r_{2}}\right) \tilde{F}_{l}^{x} \\
& +B_{l}^{n+2} \frac{r_{1} r_{2}}{\rho^{2}}\left\{F_{l}^{x+2}-\frac{\delta_{0 x}}{2}\left(F_{l}^{x+2}-\tilde{\bar{r}}_{l}^{n+2}\right)\right\}+B_{l x} \frac{r_{1} r_{2}}{\rho^{2}}\left(1-\delta_{0 x}-\delta_{1 x}\right)\left\{F_{l}^{x-2}\right. \\
& \left.\left.+\delta_{2 x} \tilde{F}_{e}^{x-2},\right\}\right]=0
\end{aligned}
$$


III THE $\mathrm{H}_{2}^{+}$IIMIT

Consider the limit in which the mass of the two identical particles becomes infinite and the third particle retains its finite mass. We shall call this the $\mathrm{H}_{2}{ }^{+}$limit even though in a real $\mathrm{H}_{2}{ }^{+}$ molecular ion the two nuclei are not infinitely heavy and the system should be described by the complete equations we have given in the previous section. Mathematically this limit is defined by

$$
m=M_{p} \rightarrow \infty
$$

$$
M=m_{e}
$$

$$
\lim _{M_{p} \rightarrow \infty} \mu=m_{e}
$$

The kinetic energy becomes

$$
\lim _{M_{p} \rightarrow \infty} T_{e}=-\frac{\hbar^{2}}{2 m_{e}}\left(\nabla_{r_{1}}^{2}+\nabla_{r_{2}}^{2}+2 \nabla_{r_{1}} \cdot \nabla_{r_{2}}\right)
$$




\section{Letting}

$$
\begin{aligned}
& \underline{m}=-\frac{1}{2}\left(\underline{m}_{1}+\underline{r}_{2}\right) \\
& \underline{r}_{12}=\underline{r}_{1}-r_{2},
\end{aligned}
$$

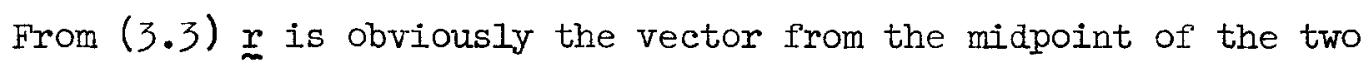
nuclei to the electron; we find for the effective kinetic energy:

$$
T_{e}=-\frac{\hbar^{2}}{2 m_{e}} \nabla_{2}^{2},
$$

so that the Schrbdirger equation becomes

$$
\left[\nabla_{r}^{2}+\frac{2 m_{e}}{\hbar^{2}}(E-V)\right] \Psi=0
$$

This is, of course, the Born-Oppenheimer approximation $\mathbf{5}^{\mathbf{5}}$ or the electronic 
motion of $\mathrm{H}_{2}^{+}$.

The potential $v$, being the attraction of the two nuclei considered as fixed centers to the electron, is not spherically symmetric.

As a result the total angular momentum of the electron is not conserved but its z-component is (the z-axis being defined as the line jolning the two nuclei). Appropriate solutions of (3.6) can therefore be written

$$
\Psi=e^{i \wedge \phi} f_{\Lambda}(r, \vartheta)
$$

where $\varphi$ and $\vartheta$ are the polar angles of $\underset{\sim}{r}$ (the vector coordinate of the electron). Substitution of (3.7) into (3.6) yields

$\left[\frac{1}{r} \frac{\partial^{2}}{\partial r^{2}} r+\frac{1}{r^{2} \sin v} \frac{\partial}{\partial v} \sin v \frac{\partial}{\partial v}-\frac{\Lambda^{2}}{r^{2} \sin ^{2} v}+\frac{2 m e}{\hbar^{2}}(E-v)\right] f_{n}=0,(3.8 a)$

where $\Lambda=0,1,2, \ldots$ correspond to the $\Sigma, \Pi, \Delta \ldots$ electronic states of $\mathrm{H}_{2}{ }^{+}$. The solutions can further be separated into even or odd electron parity $(v \rightarrow \pi-v, \varphi \rightarrow \pi+\varphi)$ corresponding to gerade and ungerade classes.

Our purpose is to derive' (3.8) from the radial equations of the 
three-body system in the $\mathrm{H}_{2}{ }^{+}$limit. This provides not only a useful check of the radial equations themselves, but it allows for the verification of general rules which relate total angular momentum states of the three-body system to their Born-Oppenheimer counterparts as one-body systems in an axially symmetric field.

We shall do this in the following way: we shall actually show for the first couple of total $\&$ radial equations in the $\mathrm{H}_{2}{ }^{+}$limit that they reduce to the form (3.8) for specific values of $\Lambda$. This will enable us to adduce a general relationship between total $\ell$ - states and the Born-Oppenheimer electronic states that they correspond to. Then we shall derive a formula relating the parity of the molecular electronic state to the parity and exchange character of the three-body wave function.

A.

$$
1=0
$$

From $(2.21)$ in the limit defined by (3.1) we have

$$
\left[L_{t / 2}^{\prime}+\frac{2 m_{e}}{\hbar^{2}}(E-V)\right] F_{0}^{0}
$$

where $\mathrm{L}_{\mathrm{r}_{12}}$ is given in (2.19). The latter is seen to depend differentially only on two coordinate: $r_{1}$ and $r_{2}$. This is already the seed of the Born-Oppenheimer limit which will be seen to apply to all $l$ equations.

$$
\text { Letting } \eta=\left|\frac{1}{2}\left(z_{1}+r_{2}\right)\right| \quad \xi=r_{12} \text { and } \rho=r_{1}^{2}-r_{2}^{2} \text {, }
$$


we find

$$
L_{r, 2}^{\prime}=\frac{2}{\eta} \frac{\partial}{\partial \eta}-\frac{\xi^{2}}{\rho^{3}} \frac{\partial}{\partial \rho}+\frac{\partial^{2}}{\partial \eta^{2}}+\frac{\rho}{\eta} \frac{\partial^{2}}{\partial \eta \partial \rho}+\frac{\xi^{2}}{\rho^{2}} \frac{\partial^{2}}{\partial \xi^{2}}
$$

Now letting $r=\eta, \cos \theta=\xi \cdot \eta / \xi \eta=\frac{\varphi^{2}}{2 \xi r}$ we find further

$$
L_{r, 2}^{\prime}=\frac{1}{r} \frac{\partial^{2}}{\partial r^{2}} r+\frac{1}{r^{2} \sin v} \frac{\partial}{\partial v} \sin v \frac{\partial}{\partial v}
$$

Thus (3.9) reduces to $(3.8)$ for $\Lambda=0$. I.e. the $l=0$ radial equation reduces to that for $\Sigma$ states in the Born-Oppenhelmer limit.

The difference between the $\Sigma$ and higher $\wedge$ equations consists of terms proportional to $\left(r^{2} \sin ^{2} \vartheta\right)^{-1}$. We need only note the relation

$$
\frac{4 r_{12}^{2}}{\rho^{2}}=\left(r^{2} \sin ^{2} v\right)^{-1}
$$


$-19-$

so that the Born-Oppenhelmer equations in general can be written

$$
\left[L_{n, 2}^{\prime}-\Lambda^{2} \frac{4 r_{12}^{2}}{\rho^{2}}+\frac{2 m e}{\hbar^{2}}(E-V)\right] f_{\Lambda}=0
$$

B. $\quad l=1$ even parity

For $\boldsymbol{l}=I$ and $\boldsymbol{x}=0, \mathrm{Eq} .(2.21)$ reduces to

$$
\left[L_{r_{12}}^{\prime}-4 \frac{r_{12}^{2}}{\rho^{2}}+\frac{2 m e}{\hbar^{2}}(E-V)\right] F_{1}^{0}=0
$$

Comparing with Eq. (3.8b) we see that this is the Born-Oppenhelmer equation for $\pi$ states.

c. $\quad l=1$ odd parity

We get from Eq. (2.20) for $\ell=1, \quad x=1$

$$
\begin{aligned}
& \because\left[L^{\prime} r_{12}+\frac{2 m e}{\hbar^{2}}(E-V)\right] F_{1}^{\prime}-\left[2 \frac{r_{12}^{2}}{\rho^{2}}+\frac{2 r_{1}^{2}+2 r_{2}^{2}-r_{12}^{2}}{4 r_{1}^{2} r_{2}^{2}}-\frac{r_{1}^{2}-r_{2}^{2}}{r_{1} r_{2} \rho}\right] F_{1}^{\prime} \\
& \quad+\left[\frac{\left(r_{1}^{2}-r_{2}^{2}\right)^{2}-r_{12}^{2}\left(r_{1}^{2}+r_{2}^{2}\right)}{r_{1} r_{2} \rho^{2}}+\frac{\left(r_{2}^{2}-r_{1}^{2}\right)\left(r_{1}^{2}+r_{2}^{2}-r_{12}^{2}\right)}{2 r_{1}^{2} r_{2}^{2} \rho}\right. \\
& \left.+\frac{\rho}{2 r_{1} r_{2}}\left(\frac{1}{r_{2}} \frac{\hat{\partial}}{\partial r_{1}}-\frac{i}{r_{1}} \frac{\partial}{\partial r_{2}}\right)\right] \tilde{F}_{1}^{\prime}=0
\end{aligned}
$$


It is also convenlent to derive a redundant equation. Ietting $r_{1} \rightleftarrows r_{2}$ in $(3.15 a)$ and using $(2.18)$, we obtain

$$
\begin{gathered}
{\left[L_{r_{12}}^{\prime}+\frac{2 m_{2}}{\hbar^{2}}(E-V)\right] \tilde{F}_{1}^{\prime}-\left[\frac{2 r_{12}^{2}}{\rho^{2}}+\frac{2 r_{1}^{2}+2 r_{2}^{2}-r_{12}^{2}}{4 r_{1}^{2} r_{2}^{2}}+\frac{r_{1}^{2}-r_{2}^{2}}{r_{1} r_{2} \rho}\right] \tilde{F}_{1}^{\prime}} \\
+\left[\frac{\left(r_{1}^{2}-r_{2}^{2}\right)^{2}-r_{12}^{2}\left(r_{1}^{2}+r_{2}^{2}\right)}{r_{1} r_{2} \rho^{2}}-\frac{\left(r_{2}^{2}-r_{1}^{2}\right)\left(r_{1}^{2}+r_{2}^{2}-r_{12}^{2}\right)}{2 r_{1}^{2} r_{2}^{2} \rho}\right. \\
\left.-\frac{\rho}{2 r_{1} r_{2}}\left(\frac{1}{r_{2}} \frac{\partial}{\partial r_{1}}-\frac{1}{r_{1}} \frac{\partial}{\partial r_{2}}\right)\right] F_{1}^{\prime}=0
\end{gathered}
$$

In order to show the Born-Oppenhelmer Ilmit of these equations we introduce the following transformation:

$$
\begin{aligned}
& F_{1}^{\prime}=H_{1}^{\prime} e^{i \alpha / 2}+i H_{-1}^{\prime} e^{-i \alpha / 2} \\
& \tilde{F}_{1}^{\prime}=-i H_{1}^{\prime} e^{i \alpha / 2}-H_{1}^{\prime} e^{-i \alpha / 2}
\end{aligned}
$$

where

$$
\tan \alpha=\frac{\left(r_{2}^{2}-r_{1}^{2}\right) p}{\left(r_{2}^{2}-r_{1}^{2}\right)^{2}-r_{12}^{2}\left(r_{1}^{2}+r_{2}^{2}\right)}
$$

This transformation has obviously not been pulled out of a hat. Rather 1t corresponds to a transformation of the Euler angle which wIIl be further discussed in the Appendix $A$.

We now make this transformation in (3.15). Multiplying the transformed of (3.15b) by $i$ and cidding to the 
transformed of (3.15a) gives

$$
\left[L_{r, 2}^{\prime}-\frac{2 r_{12}^{2}}{\rho^{2}}+\frac{2 m e}{\hbar^{2}}(E-V)\right] H_{1}^{\prime}-\frac{2 r_{12}^{2}}{\rho^{2}} H_{-1}^{\prime}=0
$$

Subtracting gives

$$
\left[L_{2,2}^{\prime}-2 \frac{r_{12}^{2}}{\rho^{2}}+\frac{2 m e}{\hbar^{2}}(E-V)\right] H_{-1}^{\prime}-2 \frac{r_{12}^{2}}{\rho^{2}} H_{1}^{\prime}=0
$$

Subtracting Eqs. (3.19a) and (3.19b), we get

$$
\left[L_{2 / 2}^{\prime}+\frac{2 m_{e}}{\hbar^{2}}(E-V)\right]\left(H_{1}^{\prime}-H_{-1}^{\prime}\right)=0
$$

which according to (3.8b) corresponds to $\Sigma$ states in the BornOppenheimer limit.

Adding Eqs. (3.19a) and (3.19b) we get

$$
\left[L_{r_{12}}^{\prime}-\frac{4 r_{12}^{2}}{\rho^{2}}+\frac{2 m e}{\hbar^{2}}(E-V)\right]\left(H_{1}^{\prime}+H_{-1}^{\prime}\right)=0
$$


to $I I$ states in the Born-Oppenheimer limit.

Therefore for $\ell=1$ we get two $\Pi$ states and one $\Sigma$ state.

D. $\quad l=2$, even parity

$\mathrm{Eq}_{\mathrm{q}}(2.21)$ in this case involves the radial functions $\mathrm{F}_{2}^{0}, \mathrm{~F}_{2}^{2}$, and $\tilde{F}_{z}^{2}$. Making the transformation

$$
\begin{array}{ll}
\mathrm{F}_{2}^{0} & =\frac{1+i}{\sqrt{2}} \mathrm{H}_{0}^{2} \\
\mathrm{~F}_{2}^{2} & =i \mathrm{H}_{2}^{2} \mathrm{e}^{i \alpha}+\mathrm{H}_{-2}^{2} \mathrm{e}^{-i_{\alpha}} \\
\tilde{\mathrm{F}}_{2}^{2} & =\mathrm{H}_{2}^{2} \mathrm{e}^{\mathrm{i}_{\alpha}}+\mathrm{iH}_{-2}^{2} \mathrm{e}^{-\mathrm{i} \alpha}
\end{array}
$$

yields three real equations for the functions $\mathrm{H}_{2}^{2}, \mathrm{H}_{-2}^{2}, \mathrm{H}_{0}^{2}$. One can readily construct linear combinations of the se equations which are the $\Sigma, \Pi$, and $\Delta$ states of $\mathrm{H}_{2}{ }^{+}$.

E. $\quad l=2$, odd parity

Here one makes the transformation

$$
\begin{array}{ll}
\mathrm{F}_{2}^{1} & =-i H_{1}^{2} e^{i_{\alpha} / 2}+H_{-1}^{2} e^{-i_{\sigma} / 2} \\
\tilde{F}_{2}^{1} & =-H_{1}^{2} e^{i \alpha / 2}+i_{-1}^{2} e^{-i \alpha / 2}
\end{array}
$$

The resultant equations can then be shown in a similar manner to be equivalent to $I$ and $\Delta$ states of $\mathrm{H}_{2}^{+}$.

Therefore, it can be shown that for $l=2$, there are $\Sigma, \pi, \Delta$ even parity states and for odd parity there are $\Pi$ and $\Delta$ states. 
This checks the wel1-known rule that for an arbitrary $\ell$ all $\Lambda$ states except $\Lambda=0$ are doubly degenerate (positive-negative) ${ }^{6}$, and for $\Sigma$ states the parity of the $l$ equation which yields this state is given by

$$
(-1)^{l+x}=1
$$

If one considers the finite mass of the nuclei, then the degeneracy of the $\Lambda \neq 0$ states is lifted giving rise to the $\Lambda$ - doubling phenomenon $^{6}$. Our results also conform the rule ${ }^{6} \Lambda \leq l$.

In order to facilitate the discussion of the $p-\mu-p$ molecule we also give the relation of the electronic gerade and ungerade classes to the three-body symmetries. This relation is

$$
i E=P E_{12}
$$

which can readily be verified two ways. First both the left and right sides have precisely the same effect on the Hamiltonian, and second if one starts with a spatial configuration of the nuclei and the electron, then the operation of $i E$ will yield the same final configuration as $P \varepsilon_{12}$.

A specific example of the above is provided by $l=0$ (there is only even parity in this case) and the $\ell=1$, odd parity cases. We have shown above the radial equations in both cases approach the $\Sigma$ 
equations of $\mathrm{H}_{2}{ }^{+}$. Nevertheless the vector spherical harmonic portions of the complete wave functions are appropriate to $\ell=0$ and $\ell=1$ respectively, and are hence different. The lowest $l=0$ wave function corresponds to the ground $\Sigma_{\mathrm{g}}$ state of $\mathrm{H}_{2}{ }^{+}$, whereas the lowest $l=1$ odd parity wave function corresponds to the first excited rotational level of the $\Sigma_{g}$ electronic state of $\mathrm{H}_{2}^{+}$. It is the latter state which in the $\mu$ mesonic counterpart of $\mathrm{H}_{2}{ }^{+}$, namely the molecule $\mathrm{p}-\mu-\mathrm{p}$, is primarily responsible ${ }^{8}$ for the absorption of $\mu$ mesons in hydrogen. The original calculation 9 which only corrects the Born-Oppenheimer approximation to the extent that the error is reduced from the order $\rightarrow$ 
$(m \mu / M p)^{1 / 4}$ to the order $\left(m_{\mu} / M p\right)$ givesa decay rate of about 560 $\sec ^{-1}$ whereas the most recent experimental number ${ }^{2}$ is $464 \pm 42 \mathrm{sec}^{-1}$. That the bulk of the discrepancy is infact due to the Born-Oppenheimer approximation has been shown by a recent calculation of Wessel and Phillipson ${ }^{3}$ who attacked the problem as a three-body problem and obtained a value of $480 \mathrm{sec}^{-1}$. Their variational calculation did in fact omit certain coupling terms in the radial equation but these omissions are of the order of $(m \mu / M p)^{2}$. (It should be noted that an accurate variational calculation has been made for this state in which all terms in the radial equations have been included ${ }^{10}$. The calculation was not primarily intended for this application.) Furthermore, we see that the ground state, $\Sigma_{g}$, is gerade $(i E=+1)$ and is derived from the $\ell=0$ equation which is necessarily even parity $(x=0)$. From $(3.28)$ it follows that $\varepsilon_{12}=+1$, so that the three-body spatial function is symmetric and its spin antisymmetric (singlet), i.e., a para state. On the other hand the capture takes place from an $\ell=1$ odd parity-state, the first rotational excited state of the $\Sigma g$ electronic state which according to (3.28) is $\varepsilon_{12}=-1$, i.e., a triplet or ortho state. Since the eigenvalue of Pis $(-1)^{x}$ we can also write

$$
i E=(-1)^{x} \varepsilon_{12}
$$




\section{APPENDIX}

This appendix is concerned with the relation between the present Euler angles and another set of symmetric Euler angles that have been Introduced ${ }^{11,12}$. This particular alternate set differs only as concerns $\Psi$. Specifically it is measured from the line of nodes to the larger of the moments of inertia in the particle plane. Letting $\Psi_{D}$ be the alternative azimuth, our objective is to find $\Psi_{D}$ as a function $\Psi$. We shail find that $\Psi_{D}$ depends additionally on $r_{1}, r_{2}$ and $\theta_{12}$, the significance of which we shall discuss further at the end of this appendix.

The alternative definition of the azimuth is given in terms of a ratio of products of inertia in a coordinate system measurea with respect to the center of mass. Letting $\xi_{i}$ be these coordinates of the three particles , we find

$$
\begin{aligned}
& \xi_{j}=\underline{\imath}_{j}+\xi_{3}, j=1,2 \\
& \xi_{3}=-\frac{1}{\mu}\left(\mu_{1} r_{j}+\mu_{2} r_{2}\right)
\end{aligned}
$$

where $\mu_{i}, i=1,2,3$, are the masses of the three particles and

$$
\mu=\sum_{i=1}^{3} \mu_{i} .
$$


$r_{j}(j=1,2)$ are the vectors from the third particle to each of the identical particles (although this analysis allows all three masses to be different.) The components of the $\xi_{i}$ are defined by

$$
\xi_{i}=\hat{i}^{\prime} \xi_{i x}+\hat{j}^{\prime} \xi_{i y}
$$

where $\hat{i}^{\prime}$ and $\hat{j}^{\prime}$ are unit vectors along $x^{\prime}$ - and $y^{\prime}-$ axes in the particle plane. We have previously given, Eqs. (II5) and (II6), $\hat{r}_{1}$ and $\hat{r}_{2}$ in the $\hat{i}^{\prime}, \hat{j}^{\prime}$ coordinate system. Substitution of these relations into (A.1) and (A.2) gives the following relations for $\xi_{i}$ :

$\xi\left(x=\left(1-\frac{\mu_{1}}{\mu}\right) r_{1} \sin \left(\Psi-\frac{1}{2} \theta_{12}\right)-\frac{\mu_{2}}{\mu} r_{2} \sin \left(\Psi+\frac{1}{2} \theta_{i 2}\right)\right.$

$\xi_{1 y}=-\left(1-\frac{\mu_{1}}{\mu}\right) r_{1} \cos \left(\Psi-\frac{1}{2} \theta_{12}\right)+\frac{\mu_{2}}{\mu} r_{2} \cos \left(\Psi+\frac{1}{2} \theta_{12}\right)$

$\xi_{2 x}=-\frac{\mu_{1}}{\mu} k_{1} \sin \left(\Psi-\frac{1}{2} \theta_{12}\right)+\left(1-\frac{\mu_{2}}{\mu}\right) r_{2} \sin \left(\Psi+\frac{1}{2} \theta_{12}\right)$.

$\xi_{2 y}=\frac{\mu_{1}}{\mu} r_{1} \cos \left(\Psi-\frac{1}{\gamma} \theta_{12}\right)-\left(1-\frac{\mu_{2}}{\mu}\right) r_{2} \cos \left(\Psi+\frac{1}{2} \theta_{12}\right)$ 
$\varepsilon_{3 x}=-\frac{\mu_{1}}{\mu} r_{1} \sin \left(\Psi-\frac{1}{2} \theta_{12}\right)-\frac{\mu_{2}}{\mu} r_{2} \sin \left(\Psi+\frac{1}{2} \theta_{12}\right)$

$\xi_{3 y}=\frac{\mu_{1}}{\mu} r_{1} \cos \left(\bar{\psi}-\frac{1}{2} \theta_{12}\right)+\frac{\mu_{2}}{\mu} r_{2} \cos \left(\Psi+\frac{1}{2} \theta_{12}\right)$

The defintion of $\Psi_{D}$ is given $b^{13}$

$$
\tan 2 \Phi_{0}=\frac{-2 D_{x y}}{D_{x x}-D_{y y}}
$$

where

$$
\begin{aligned}
D_{x y} & =\sum_{i=1}^{3} \mu_{i} \xi_{i x} \xi_{i y} \\
D_{x x} & =\sum_{i=1}^{3} \mu_{i} \xi_{i y}^{2} \\
D_{y y} & =\sum_{i=1}^{3} \mu_{i} \xi_{i x}^{2}
\end{aligned}
$$

Substituting $(A .5)-($ A.10) into $(A .12)-(A .14)$ yields 


$$
\begin{aligned}
\tan 2 \Phi_{D}= & {\left[\mu_{1}\left(1-\frac{\mu_{1}}{\mu}\right) r_{1}^{2} \sin \left(2 \Psi-\theta_{12}\right)+\mu_{2}\left(1-\frac{\mu_{2}}{\mu}\right) r_{2}^{2} \sin \left(2 \Phi+\theta_{12}\right)\right.} \\
& \left.-\frac{2 \mu_{1} \mu_{2}}{\mu} r_{1} r_{2} \sin 2 \Psi\right]\left[\mu_{1}\left(1-\frac{\mu_{1}}{\mu}\right) \mu_{1}^{2} \cos \left(2 \Phi-\theta_{12}\right)\right. \\
& \left.+\mu_{2}\left(1-\frac{\mu_{2}}{\mu}\right) r_{2}^{2} \cos \left(2 \Psi+\theta_{12}\right)-\frac{2 \mu_{1} \mu_{2}}{\mu} r_{1} k_{2} \cos 2 \Psi\right]^{-1}
\end{aligned}
$$

Specializing to $\mathrm{H}_{2}{ }^{+}$Iimit, we have

$$
\begin{aligned}
& \mu_{2}=\mu_{2}=M_{p} \rightarrow \infty \\
& \mu_{3}=m_{e} \\
& \tan 2 \Psi_{D}=\frac{\left[\left(r_{1}^{2}+r_{2}^{2}\right) \cos \theta_{12}-2 r_{1} r_{2}\right] \sin 2 \Psi+\left(r_{2}^{2}-r_{1}^{2}\right) \sin \theta_{12} \cos 2 \Psi}{\left(r_{1}^{2}-r_{2}^{2}\right) \sin \theta_{12} \sin 2 \Psi+\left[\left(r_{1}^{2}+r_{2}^{2}\right) \cos \theta_{12}-2 r_{12}\right] \cos 2 \Psi} \\
&= \\
& \tan (2 \Psi+\alpha),
\end{aligned}
$$




$$
\tan \alpha=\frac{\left(n_{2}^{2}-x_{1}^{2}\right) \sin \theta_{12}}{\left(i_{1}^{2}+n_{2}^{2}\right) \cos \theta_{12}-2 k_{1} n_{2}} .
$$

Using

$$
\cos \theta_{12}=\frac{r_{1}^{2}+r_{2}^{2}-r_{12}^{2}}{2 r_{1} r_{2}},
$$

we find

$$
\tan \alpha=\frac{\left(r_{2}^{2}-r_{1}^{2}\right) \rho}{\left(r_{1}^{2}-r_{2}^{2}\right)^{2}-r_{12}^{2}\left(r_{1}^{2}+r_{2}^{2}\right)}
$$

With the relation (A.16) one can then transform the wave function 12

$$
\Psi=\sum_{k=-l}^{l} H_{k}^{l}\left(r_{1}, r_{2}, k_{12}\right) \delta_{l}^{m, k}\left(\theta, \Phi, \Psi_{3}\right)
$$

Into our form Eq. (2.14), from which one derives the relation between 
$f_{l}^{x \pm}$ (and hence $F_{l}^{x}$ ) and $\mathrm{H}_{k}^{\ell}$.

The radial equations which result are consequently precisely those that one would get directly from the formulae of Diehl et. al. 9 in the $\mathrm{H}_{2}{ }^{+}$limit. The simplicity of their equations from the point of view of this limit stems from the fact that the axis to which W is measured becomes identical with the line joining the nuclei, i.e., the z-axis, in the Born-Oppenheimer approximation.

In the case of all finite masses, however, there arises an additional group of terms in their equations. A comparison with our equations indicates that in general their equations are more complicated than ours. In particular some of the coefficlents have a more complicated analytic behavior. We believe this is related to an argument in I which stated that because our Euler angles depend only on the unit vectors $\hat{r}_{1}$ and $\hat{r}_{2}$ and not on the magnitudes of $r_{1}$ and $r_{2}$, we think our Euler angles are supertor; for they retain the separation between angular and radial parts which was implicit in the expansion of the complete wave function itself (cf. (2.14) and $(A .20))$. The suggestion that we are making here is that a concrete manifestation of such a superiority may reside precisely in the differences in the analytic structure of the respective radial equations.

The dependence of $\Psi_{3}$ on $r_{1}$ and $r_{2}$ is explicitly exhibited in Eqs. (A.16) and (A.17). In Appendix III of reference 1 we derived the relation between ours and the Hylleraas-Breit Euler angles 14,15. 
The latter have the disadvantage of not being symmetrical, but they too depend only on the unit vectors $\hat{r}_{1}$ and $\hat{r}_{2}$. Therefore the relations between the respective Euler angles are independent of $r_{1}$ and $r_{2}$. According to the above argument we do not expect any significant difference in the analytic properties of Breit's (P state) radial equations ${ }^{15}$ as compared to our own. 
Appendix B

- For three unequal mass particles, the kinetic energy in the center of mass system is

$$
T=-\frac{1}{2 \mu_{1}} \nabla_{n_{1}}^{2}-\frac{1}{2 \mu_{2}} \nabla_{r_{2}}^{2}-\frac{1}{M} \nabla_{n_{1}} \cdot \nabla_{n_{2}}
$$

where

$$
\begin{aligned}
& \mu_{1}=\frac{m_{1} M}{m_{1}+M} \\
& \mu_{2}=\frac{m_{2} M}{m_{2}+M}
\end{aligned}
$$

The Schrödinger equation in the c.m. system is thus

$$
\left[\nabla_{r_{1}}^{2}+\mu_{1 / \mu_{2}} \nabla_{r_{2}}^{2}+\frac{2 \mu_{1}}{M} \nabla_{n_{1}} \cdot \nabla_{r_{2}}+\frac{2 \mu_{1}}{\hbar^{2}}(E-V)\right] \Psi=0 .
$$

It has been shown how the individual Laplacian may be written in terms of the Euler angles and residual coordinates $r_{1}, r_{2}, \theta_{12}$ :

$$
\nabla_{r_{1}}^{2}=\frac{1}{r_{1}} \frac{\partial^{2}}{\partial r_{1}^{2}} r_{1}+\frac{1}{r_{1}^{2}}\left\{\frac{1}{\sin \theta_{12}} \frac{\partial}{\partial \theta_{12}} \sin \theta_{12} \frac{\partial}{\partial \theta_{12}}+F_{1}(\theta, \Phi, \psi)\right\} \text { (B.5) }
$$


$\nabla_{r_{2}}^{2}=1 / r_{2} \frac{\partial^{2}}{\partial r_{2}^{2}} r_{2}+\frac{1}{r_{2}^{2}}\left\{1 / \sin \theta_{12} \frac{\partial}{\partial \theta_{12}} \sin \theta_{12} \frac{\partial}{\partial \theta_{12}}+F_{2}(\theta, \Phi, \psi)\right\}$

where $F_{1}$ and $F_{2}$ are given in Eqs. (I59, I62). Knowing then the effect of $F_{1}, F_{2}$ and the cross term $\quad \nabla_{\mu_{1}} \cdot \nabla_{\mu_{2}}$ on the vector spherical harmonics via their representation in terms of the $\Lambda_{1}, \Lambda_{2}$ nerators in $(163,64)$ and $(2.13)$, one may readily derive the radial equations for the general case. As coupled equations in terms of the residual coordinates $\boldsymbol{r}_{1}, \boldsymbol{r}_{2}, \theta_{12}$, the radial equations are obtained by making the following substitution in (2.15):

$$
\mu \rightarrow \mu_{1}
$$

$r_{1}^{-2} \pm r_{2}^{-2} \rightarrow r_{1}^{-2} \pm \mu_{1} / \mu_{2} r_{2}^{-2}$

$L_{\theta_{12}} \rightarrow \bar{L}_{\theta_{12}}=\frac{1}{r_{1}} \frac{\partial^{2}}{\partial r_{1}^{2}} r_{1}+\mu_{1 / \mu_{2}} \frac{1}{r_{2}} \frac{\partial^{2}}{\partial r_{2}^{2}} r_{2}$

$$
+\left(\frac{1}{r_{1}^{2}}+\frac{\mu_{1}}{\mu_{2}} \frac{1}{r_{2}^{2}}\right) \frac{1}{\sin \theta_{12}} \frac{\partial}{\partial \theta_{12}} \sin \theta_{12} \frac{\partial}{\partial \theta_{12}}
$$

Since for $\mathrm{m}_{1} \neq \mathrm{m}_{2}$ the equations are no longer symmetric with respect to $r_{1} \rightleftarrows r_{2}$, it is necessary to solve the equations for all values of $r_{1}$ 
$-35-$

and $r_{2}$ (as opposed to, say, $r_{1} \geqslant r_{2}$ and an appropriate boundary condition when $m_{1}$ is identical to $m_{2}$ ). By virtue of this there is no advantage in defining functions $F_{l}^{*}$ and $\widetilde{F}_{l}^{\mu}$ as they will also be described by a coupled set of equations, rather than a single equation (2.2l) for the identical particle case. For this reason we give below the equations in terms of the coupled. $f_{l}^{x \pm}$ functions, but involving the coordinates $r_{1}, r_{2}, r_{12} \cdot$

$$
\begin{aligned}
& {\left[\bar{L}_{n, 2}+\frac{2 \mu_{1}}{\hbar^{2}}(E-V)\right] f_{l}^{x+}-\left(\frac{1}{n_{1}^{2}}+\frac{\mu_{1}}{\mu_{2}} \frac{1}{n_{2}^{2}}\right)\left[\left\{\left(l(l+1)-x^{2}\right) \frac{2 n_{1}^{2} x_{2}^{2}}{\rho^{2}}\right.\right.} \\
& \left.+\frac{x^{2}}{4}-\frac{r_{1} r_{2}}{2 p^{2}}\left(r_{1}^{2}+r_{2}^{2}-r_{12}^{2}\right) \delta_{1 n} l(l+1)\right\} f_{l}^{x+} \\
& \left.+\frac{r_{1} r_{2}}{2 \rho^{2}}\left(r_{1}^{2}+r_{2}^{2}-r_{12}^{2}\right)\left\{B_{l}^{x+2} f_{l}^{(x+2)+}+\left(1-\delta_{0 x}-\delta_{1 x}+\delta_{2 x}\right) B_{l} x f_{l}^{(x-2)+1}\right\}\right] \\
& +\left(\frac{1}{r_{1}^{2}}-\frac{\mu_{1}}{\mu_{2}} \frac{1}{r_{2}^{2}}\right)\left[-\left\{\frac{x}{2}\left(\frac{r_{1}^{2}+r_{2}^{2}-r_{12}^{2}}{\rho}+\frac{\rho}{r_{12}} \frac{\partial}{\partial r_{12}}\right)\right.\right. \\
& \left.+l(l+1) \delta_{1 x} \frac{z_{1} r_{2}}{2 \rho}\right\} f_{l}^{x-}+\frac{z_{1} r_{2}}{2 \rho}\left\{B_{l}^{x+2} f_{l}^{(x+2)-}\right. \\
& -\left(1-\delta_{0 x}-\delta_{1 x}-\delta_{2 x}\right) B_{R x} f_{l}^{(x-2)-\}]} \\
& +\frac{2 \mu_{1}}{m_{3}}\left[\left\{\left(r_{1}^{2}+r_{2}^{2}-r_{12}^{2}\right)\left(\frac{\ell(\rho+1)-x^{2}}{\rho^{2}}-\frac{x^{2}}{8 r_{1}^{2} r_{2}^{2}}\right)-l(l+1) \delta_{1 x} \frac{r_{1} r_{2}}{\rho^{2}}\right\} f_{l}^{x+}\right. \\
& +\frac{z_{1,2}}{\rho^{2}}\left\{B_{l}^{x+2} f_{l}^{(x+2)+}+\left(1-\delta_{x}-\delta_{1 x}+\delta_{2 x}\right) f_{l}^{(x-2)+}\right\}
\end{aligned}
$$


$-30-$

$$
\begin{aligned}
& \left.+\frac{x \rho}{4 r_{1} r_{2}}\left(\frac{1}{r_{1}} \frac{\partial}{\partial r_{2}}-\frac{1}{r_{2}} \frac{\partial}{\partial r_{1}}+\frac{r_{2}^{2}-r_{1}^{2}}{r_{1} r_{2}} \frac{1}{r_{12}} \frac{\partial}{\partial r_{12}}\right) f_{l}^{x-}\right]=0 \\
& {\left[\bar{L}_{r 12}^{\prime}+\frac{2 \mu_{1}}{\hbar^{2}}(E-V)\right] f_{l}^{x-}-\left(\frac{1}{r_{1}^{2}}+\frac{\mu_{1}}{\mu_{2}} \frac{1}{r_{2}^{2}}\right)\left[\left\{\left(l(l+1)-x^{2}\right) \frac{2 r_{1}^{2} r_{2}^{2}}{\rho^{2}}\right.\right.} \\
& \left.+\frac{n^{2}}{4}+\frac{r_{1} r_{2}}{2 \rho^{2}}\left(r_{1}^{2}+r_{2}^{2}-r_{12}^{2}\right) \delta_{1 x} l(l+1)\right\} f_{l}^{x-} \\
& +\frac{r_{1} r_{2}}{2 \rho^{2}}\left(r_{1}^{2}+r_{2}^{2}-r_{12}^{2}\right)\left\{\left(1-\delta_{0 x}\right) B_{l}^{x+2} f_{l}^{(x+2)-}+\left(1-\delta_{0 x}-\delta_{1 x}-\delta_{2 x}\right) B_{l x} f_{l}^{(x-2)-\}}\right\} \\
& +\left(\frac{1}{r_{1}^{2}}-\frac{\mu_{1}}{\mu_{2}} \frac{1}{r_{2}^{2}}\right)\left[\left\{\frac{x}{2}\left(\frac{r_{1}^{2}+r_{2}^{2}-r_{12}^{2}}{\rho}+\frac{\rho}{r_{12}} \frac{\partial}{\partial r_{12}}\right)-l(l+1) \delta_{1 x} \frac{r_{1} r_{2}}{2 \rho}\right\} f_{l}^{x+}\right. \\
& \left.-\frac{r_{1} r_{2}}{2 \rho}\left\{\left(1-\delta_{0 x}\right) B_{l}^{x+2} f_{l}^{(x+2)+}-\left(1-\delta_{0 x}-\delta_{1 x}+\delta_{2 x}\right) B_{l x} f_{l}^{(x-2)+}\right\}\right] \\
& +\frac{2 \mu_{1}}{m_{3}}\left[\left\{\left(r_{1}^{2}+r_{2}^{2}-r_{12}^{2}\right)\left(\frac{\ell(\rho+1)-x^{2}}{\rho^{2}}-\frac{x^{2}}{8 r_{1}^{2} r_{2}^{2}}\right)+l(l+1) \delta_{1} \times \frac{r_{1} r_{2}}{\rho^{2}}\right\} f_{l}^{x-}\right. \\
& +\frac{r_{1} \lambda_{2}}{\rho^{2}}\left\{\left(1-\delta_{0 x}\right) B_{l}^{x+2} f_{l}^{(x+2)-}+\left(1-\delta_{0 x}-\delta_{1 x}-\delta_{2 x}\right) B_{l x} f_{l}^{(x-2)-}\right\} . \\
& \left.+\frac{x \rho}{4 r_{1} r_{2}}\left(\frac{1}{r_{2}} \frac{\partial}{\partial r_{1}}-\frac{1}{r_{1}} \frac{\partial}{\partial r_{2}}+\frac{r_{1}^{2}-r_{2}^{2}}{r_{1} r_{2}} \frac{1}{r_{12}} \frac{\partial}{\partial r_{12}}\right) f_{l}^{x+}\right]=0
\end{aligned}
$$

where

$$
\begin{aligned}
\bar{L}_{r_{12}}= & \frac{1}{r_{1}} \frac{\partial^{2}}{\partial r_{1}^{2}} r_{1}+\frac{\mu_{1}}{\mu_{2}} \frac{1}{r_{2}} \frac{\partial^{2}}{\partial r_{2}^{2}} r_{2}+\frac{\mu_{1}}{\mu_{12}} \frac{1}{r_{12}} \frac{\partial^{2}}{\partial r_{12}^{2}} r_{12}+\frac{\mu_{1}}{m_{1}} \frac{r_{1}^{2}+r_{12}^{2}-r_{2}^{2}}{r_{1} r_{12}} \frac{\partial^{2}}{\partial r_{1} r_{12}} \\
& +\frac{\mu_{1}}{m_{2}} \frac{r_{2}^{2}+r_{12}^{2}-r_{1}^{2}}{r_{2} r_{12}} \frac{\partial^{2}}{\partial r_{2} \partial r_{12}}+\frac{\mu_{1}}{m_{3}} \frac{r_{1}^{2}+r_{2}^{2}-r_{12}^{2}}{r_{1} r_{2}} \frac{\partial^{2}}{\partial r_{1} \partial r_{2}} \quad \text { (B. I2) }
\end{aligned}
$$


and

$$
\mu_{12}=\frac{m_{1} m_{2}}{m_{1}+m_{2}}
$$

The resulting radial equations are invarlant (except for a relative phase factor which does not affect the eigenvalue spectrum) under the simultaneous exchange of $\mu_{1} \vec{a} r_{2}$ and $m_{1} \vec{m} m_{2}$. However the equations are not (formally) invariant under a simultaneous permutation of all three particles (1.e., simultaneous cyclic permutations of $m_{1}, m_{2}$, $m_{3}$ and $\left.r_{1}, r_{2}, r_{12}\right)$. This is apparently due to the fact that our choice of Euler angles singles out one particle, the particle with mass $M$, as the instantaneous origin. This in turn means that the line to which the azimuth $\Psi$ is measured depends asymmetrically on which particle we call the origin. As opposed to this the Euler angles of Holmberg ${ }^{I l}$ and Diehl et al. ${ }^{12}$ are invariant under the operation, and their radial equations are also.

Since, however, the transformation between the respective radial functions can be worked out from Eq. (A.15), this additional symmetry can readily be recovered. It would seem that a more practical criterion of the utility of the equations is the analytic propertles of the equations themselves. In this respect the discussion near the end of Appendix A may be more relevant. 


\section{ACKONOWIEDGEMMENIS}

The analysis of Section III was undertaken as a direct result of work of $C$. W. Scherr and M. Machacek on the P-states of three-body systems Including the $\mathrm{H}_{2}^{+}$IImft (cf. reference10). At the inception of these considerations, Dr. A. Dalgarno was a visitor with the Theoretical Division; we should like to thank Dr. Dalgamo for valuable discussions in this connectlon. Thanks are also due to Dr. Richard Drachman for discussions. The ( $p-\mu-p)$ problem was brought to our attention by $\mathrm{Dr}$. Arnold Tubis. 


\section{REFERENCES}

+National Academy of Sciences - National Research Council Resident Research Associate

1. A. K. Bhatia and A. Temkin, Rev. Mod. Phys. 36, (1964), and Goddard Space Flight Center preprint X 640-64-62 (May 1964, unpublished.) This will be referred to $I$ and equations referring to it will be prefixed by $I$.

2. J. E. Rothberg, E. W. Anderson, E. J. Bleser, L. M. Lederman, S. L. Meyer, J. L. Rosen, I-T Wang, Phys. Rev. 132, 2664 (1963).

3. W. Roy Wessel and P. Phillipson, Phys. Rev. Letters 13, 23 (1964).

4. C. L. Pekeris, Phys. Rev. 112, 1649 (1958) and subsequent papers.

5. M. Born and J. R. Oppenheimer, Ann. Physik 8녀, 457 (1927).

6. G. Herzberg, Spectra of Diatomic Molecules (Van Nostrand Inc., New York, 1963).

7. The relation $(3.28)$ is equivalent to the identity of the parity of the rotational part of the wave function and exchange of the nuclei. As such it has been known at least since the work of D. M. Dennison, Proc. Roy. Soc. (London) 115, 483 (1927), in his invocation of the ortho-para idea to explain the specific heat of $\mathrm{H}_{2}$.

8. S. Weinberg, Phys. Rev. Letters 4, 575 (1960).

9. S. Cohen, D. L. Judd, and R. J. Ridde11, Phys. Rev. 119, 384 , 397 (1960).

10. C. W. Scherr and M. Machacek, Bulletin of the American Physical Society, Series II $9,230(1964)$ and (to be published). 
11. B. Holmbcre, Kungl. Fysiografiska Sbllskapets I Lund FBrhandiingar ef, 1 (1956)。

12. Ii. Diehl, S. Fl'ts, U. Schroder, A. VHIke1, A. Weiguny, Z. Physik 16e, I (196]). In order for the exchange property (3.36) to be comletiy consistent, the a functions in

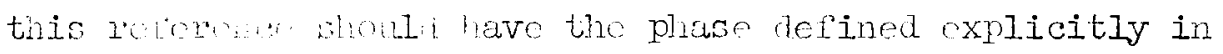
I.PIUne 1 .

13. We should Lik io whin Dr. Peter Muon for helping us to understian this ionitidu.

14. F, A. Hyllerias, Z. Physir 48,469 (1928)。

15. Go Brict, Phys. Rev. 35, 569 (1930). 
TABIE I

Coefficients of the Angular Derivatives in the Cross Term of the Kinetic Energy. ${ }^{a}$

$\cdots+$

\begin{tabular}{|c|c|c|c|}
\hline $\begin{array}{l}\text { Coefficient } \\
-\cos \theta_{12}\end{array}$ & $\left\{\begin{array}{l}\text { Derivative } \\
\frac{\partial^{2}}{\partial \theta_{12}}\end{array}\right.$ & $\begin{array}{l}\text { Coefficient } \\
\frac{-\cot \theta \sin 2 \Psi}{\sin ^{2} \theta_{12}}\end{array}$ & $\begin{array}{l}\text { Derivative } \\
\frac{\partial^{2}}{\partial \theta \partial \Psi}\end{array}$ \\
\hline$\frac{\cos 2 \Psi-\cos \theta_{12}}{2 \sin ^{2} \theta_{12}}$ & $\frac{\partial^{2}}{\partial \theta^{2}}$ & $\frac{\cos \theta\left(\cos \theta_{12}+\cos 2 \Psi\right)}{\sin ^{2} \theta \sin ^{2} \theta_{12}}$ & $\frac{\partial^{2}}{\partial \Psi \partial \Phi}$ \\
\hline$-\sin \theta_{12}$ & $\frac{\partial^{2}}{\partial r_{(}}\left(\begin{array}{l}1 \\
)^{\theta_{12}}\end{array}\right.$ & $\frac{\cos \theta_{12}}{4}-\frac{\cot ^{2} \theta}{2 \sin ^{2} \theta_{12}}\left(\cos \theta_{12}+\cos 2 \psi\right)$ & $\frac{\partial^{2}}{\partial y^{2}}$ \\
\hline$-\frac{1}{2} \sin \theta_{12}$ & $\frac{\partial^{2}}{\partial r_{1} \partial \psi}$ & $-\frac{1}{\sin \theta_{12}}$ & $\frac{\partial}{\partial \theta_{12}}$ \\
\hline$+\frac{1}{2} \sin \theta_{12}$ & $\frac{\partial^{2}}{\partial r_{2} \partial \Psi}$ & $\frac{-\cot \theta}{2 \sin ^{2} \theta_{12}}\left(\cos 2 \Psi+\cos \theta_{12}\right)$ & $\frac{\partial}{\partial \theta}$ \\
\hline
\end{tabular}

$\frac{\sin 2 \Psi}{\sin ^{2} \theta_{12} \sin \theta} \quad \frac{\partial^{2}}{\partial \theta \partial \Phi} \quad \frac{-\cot \theta \sin 2 \psi}{\sin \theta \sin ^{2} \theta_{12}}$

4 .

$-\frac{\left(\cos \theta_{12}+\cos 2 \Psi\right)}{2 \sin ^{2} \theta_{12} \sin ^{2} \theta} \frac{\partial^{2}}{\partial \Phi^{2}}$

$\frac{\sin 2 \Psi}{2 \sin ^{2} \theta_{12}}\left(1+2 \cot ^{2} \theta\right)$

$\frac{\partial}{\partial \Psi}$

athe dependence on $r_{1}$ and $r_{2}$ is not included. All unincluded partial derivatives have zero coefficients. 